\title{
Guest Editor Note
}

\author{
Lisa Miller ${ }^{1}$
}

Published online: 9 April 2015

(C) Springer Science+Business Media New York 2015

I am delighted and honored that the Editor-in-Chief of The Journal of Religion and Health, Curtis Hart, has welcomed a Special Section "Spirituality in Adolescents: The Hub of Mental Health and Positive Development." JRH engages the thoughtful and reflective readership that I hope will join in discussion around the science in this Special Section. To share a sense of location, I write to you from the Spirituality Mind Body Institute at Columbia University, Teachers College. Here, we conduct research, practice, and train graduate students in foundationally spiritual perspectives.

Over the past 15 years, a body of research has painted a clear picture that spirituality is the hub of wellness and thriving of adolescents. The research in brief summary shows that we are inherently spiritual beings, with an innate capacity for transcendence and a capacity to form a dynamic relationship with a Higher Power. In adolescence comes a biologically supported window of "spiritual awakening," to include a surge in the transcendent capacity, and a driving myriad of questions of ultimate purpose and meaning. Science here shows that which world religions have supported through millennium and is reflected in the global traditions of puberty rites. Our society omits mention and support of spiritual development to the peril of youth. Often I hear practicing therapists and clergy share accounts of teens who ask the "big questions," and whose behavior inciting treatment is a misguided attempt to feel transcendence and spiritual communion.

Research consistently shows that a strong personal spirituality (primarily a direct relationship with a Higher Power, Universe, G-d, Allah, Hashem) is highly protective against prevalent forms of suffering, recurrent depression, substance abuse, and risk taking. This personal relationship with the Higher Power also is foundational in adolescence to wellbeing and positive psychology traits. Neighboring adolescents can live on the same block, attend the same schools, and confront the same peers each day. Yet the adolescent with a strong personal relationship with the Higher Power generally is $40 \%$ less likely to be a

Lisa Miller

1fm14@columbia.edu

1 Spirituality Mind Body Institute, Teachers College, Columbia University, New York, NY, USA 
substance abuser, $60 \%$ less likely to have recurrent depression, and $80 \%$ less likely to have risky unprotected sex.

The most crucial work of adolescence is spiritual individuation. We can support spiritual individuation in adolescents as mental health providers, clergy, community, and family. And if we do, the adolescent builds a spiritual foundation that sustains a healthier and more thriving adult life.

The current Special Section advances the research on spirituality in adolescence by examining the presentation and pathway of the development of a spiritual hub, as well as some of its multiple effects. The spiritual hub in adolescence is observed both in suffering with depression and in the face of thriving.

The most prevalent form of suffering is depression, as moderate subthreshold depression affects over $40 \%$ of adolescents, beyond the 20-25\% to have MDD. Together with my collaborator, I explore a frequent presentation, Developmental Depression, linked to the emergence of transcendence, for unique neural correlates and rates of comorbid symptoms. Turning to a study of spirituality and thriving, Yakov Barton and colleagues show in large sample of adolescents that spirituality and positive psychology traits go hand in hand. Namely, four or five adolescents are as relatively strong in positive psychology traits (as measured by optimism, grit, commitment, forgiveness) as they are engaged with a Higher Power.

The formation of the spiritual hub in adolescents is explored through three articles on spiritual supports and pathways of development. Eleanor Cobb and colleagues reveal in early adolescents the unique contributions of both religious practice and trait mindfulness on spirituality. In a longitudinal data set, Sam Barkin and colleagues investigate the longterm stability of adolescent spirituality. The level of personal spirituality by senior year in high school is examined vis-a-vis level of spirituality six years later, two years postcollege, when the emerging adult now lives independently. Taking a broad view of development in later adolescence and emerging adulthood, Peter Sanders, Scott Richards, and colleagues examine the role of intrinsic spirituality in an observant religious community, The Church of Latter-Day Saints, on processes of identity integration, moral self-approval, and meaning in life, along with a host of mental health benefits.

A spiritually based treatment may support the formation of a spiritual hub for adolescents, which in turn has broad benefits. In an open clinical trial, Dinesh Braganz and Ralph Piedmont show that "Core Training" as means to address the spiritual opportunity in a broad range of current struggles is equally feasible and equally helpful, when done by adolescents as by adults. Among youth with extreme struggles, homeless youth, Biagio Mastriopieri and colleagues report on a shelter-based spiritual intervention that focuses on the integration of spirituality into daily relationships. Together with interpersonal work, a spiritual visualization practice conducted in group was shown to ignite a sense of a sacred self and sacred fellow being, as well as benefit mental health and self-reliance.

The spiritual hub is "command central" for the adolescent, informing all other lines of social, emotional, and moral development. Together these articles converge to show that the spiritual hub can be supported by psychotherapists, clergy, family, and community, to the great benefit of the adolescent now and into adulthood. 